\title{
Dock2 affects the host susceptibility to Citrobacter rodentium infection through regulating gut microbiota
}

Yuan Xie ${ }^{1,2}$, Jun Chen², Bing Wü ${ }^{3}$ Tiansheng $\mathrm{He}^{3}$, Lu Xie ${ }^{3}$ and Zhiping $\mathrm{Liu}^{3,4^{*}}$ (D)

\begin{abstract}
Background: Dysregulated gut microbiota is one of major pathogenic factors in the development of colitis. Dock2 acts as a guanine nucleotide exchange factor (GEF) and activates small G protein RAC1. Our previous study showed that, compared to wild type (WT) mice, Dock $2^{-1-}$ mice were more susceptible to colitis induced by Citrobacter rodentium infection. However, it is not clear whether gut microbiota affects the host susceptibility to enteric bacterial infection in Dock2 ${ }^{-/}$mice.

Results: In this study, we demonstrated that Dock2 regulated the gut microbiota and affected the host susceptibility to C. rodentium infection by co-housing, fecal microbiota transfer and antibiotic treatment methods. Microbiota analysis by $16 \mathrm{~S}$ rRNA gene sequencing showed that Dock2 increased the abundance of prevotellaceae-NK3B31-group and Lactobacillus but decreased that of Helicobacter.
\end{abstract}

Conclusions: These results suggest that Dock2 regulates the composition of gut microbiota and affects the host susceptibility to $C$. rodentium infection.

Keywords: Gut microbiota, Dock2, Citrobacter rodentium, Colitis, 16S rRNA gene sequencing

\section{Introduction}

Inflammatory bowel disease (IBD), including Crohn's disease (CD) and ulcerative colitis (UC), causes chronic relapsing inflammatory disorders in genetically susceptible individuals $[1,2]$. The pathogenesis of IBD involves dysregulation of gut microbiota, genetic susceptibility and immune abnormalities. Gut microbiota plays a crucial role in regulating host responses to pathogens and maintaining intestinal homeostasis. Dysregulated gut microbiota may suppress mucosal immune system, damage intestinal epithelial cells, increase the intestinal permeability, thus inducing intestinal inflammation [3].

*Correspondence: Zhiping.Liu@gmu.edu.cn

${ }^{3}$ School of Basic Medicine, Gannan Medical University, Jiangxi, Ganzhou China

Full list of author information is available at the end of the article
However, the exact pathogenesis of IBD is still unclear. IBD animal model is an important means to study the pathogenesis. Among them, Citrobacter rodentium infection is often used to study the formation of colitis [4]. C. rodentium is a gram-negative bacterium in the intestine, which can induce intestinal proliferation and inflammation. Different from another commonly used colitis model which is induced by innate immune response by the treatment of dextran sodium sulfate, $C$. rodentium infection induces host immune response involving with both innate and adaptive immunity, which is more similar to IBD patients [4].

Dedicator of cytokinesis 2 (Dock2) is a guanine exchange factor (GEFs), which specifically activates the small G protein RAC1 by mediating GTP-GDP exchange and regulates the formation of cytoskeleton [5]. Our previous study showed that Dock $2^{-/-}$mice were more susceptible to colitis induced by C. rodentium infection, original author(s) and the source, provide a link to the Creative Commons licence, and indicate if changes were made. The images or other third party material in this article are included in the article's Creative Commons licence, unless indicated otherwise in a credit line to the material. If material is not included in the article's Creative Commons licence and your intended use is not permitted by statutory regulation or exceeds the permitted use, you will need to obtain permission directly from the copyright holder. To view a copy of this licence, visit http://creativecommons.org/licenses/by/4.0/. The Creative Commons Public Domain Dedication waiver (http://creativeco mmons.org/publicdomain/zero/1.0/) applies to the data made available in this article, unless otherwise stated in a credit line to the data. 
which was mainly manifested by higher mortality, weight loss, C. rodentium load and intestinal damage [6]. However, the precise mechanism by which Dock2 regulates the host susceptibility to $C$. rodentium infection remains largely unclear.

Numerous studies suggested that microbiota composition was critical for host resistance to $C$. rodentium infection [7]. Compared to conventional mice, germ-free mice could not clear the enteric pathogen at late stage of C. rodentium infection [8]. The commensal Escherichia coli, but not Bacteroides species, could outcompete luminal $C$. rodentium by utilizing metabolites such as monosaccharides [8]. The probiotic Lactobacillus, either used alone or with other probiotics, could inhibit the intestinal inflammation induced by $C$. rodentium infection through inducing the production of IL-22 and activating regulatory T cells [9]. Segmented filamentous bacteria (SFB), an anaerobic commensal bacterium tightly adherent to intestinal epithelial cells, enhanced the host resistance to $C$. rodentium infection by inducing intestinal Th17 cells $[10,11]$. The abundance of Lachnospiraceae, one of major short-chain fatty acid-producing commensal bacteria, was correlated with less intestinal inflammation induced by $C$. rodentium infection [12]. Administration of Clostridiales conferred the host protection to $C$. rodentium infection in neonatal mice that lack Clostridiales while depletion of Clostridiales abolished colonization resistance in adult mice, indicating that Clostridiales enhanced host resistance to $C$. rodentium infection [13]. Under fiber-deprived diet, mucus-degrading commensals, such as Akkermansia muciniphila, could shift from metabolizing dietary polysaccharides to degrading mucus glycan, thus damaging mucus barrier and increasing host susceptibility to C. rodentium infection [14]. However, the roles of Dock2 in the regulation of gut microbiota and host susceptibility to $C$. rodentium infection are unknown.

Here, we tested the impact of Dock2 on the composition of gut microbiota and the host susceptibility to $C$. rodentium infection by co-housing, gut microbiota transfer and antibiotic treatment experiments. Moreover, we detected the specific gut microbiota changes by $16 \mathrm{~S}$ rRNA sequencing. This study elucidated the effects of gut microbiota on host susceptibility of Dock $2^{-/-}$mice to $C$. rodentium infection and provided a new theoretical basis for the treatment of intestinal inflammatory diseases.

\section{Materials and methods}

\section{Mice}

Wild-type (WT) and Dock2 $2^{-/}$mice on C57BL/6 background were provided by Yoshinori Fukui (Kyushu University, Japan). Mice were housed in SPF environment of Experimental Animal Center of Gannan Medical
University. The lights were adjusted to simulate normal day and night, and mice had ad libitum access to sterile drinking water. The 4 to 6 week-old mice were selected. Except that the mice selected for the cohousing experiment were female mice to prevent fighting against each other, the other mice were either female or male mice(Same gender in the same cage). This study was approved by the Ethics Committee of Gannan Medical University.

\section{Co-housing experiment}

The cohousing experiment methods were performed as previously described with modification [15]. Specifically, female mice were used for cohousing experiment to prevent the fighting against each other. WT and Dock $2^{-/-}$ mice were housed in the same cages for 4 weeks. After that, the mice were separated into different cages according to their genotypes and then infected with $C$. rodentium. Fecal samples of mice before and after co-house were collected and stored at $-80{ }^{\circ} \mathrm{C}$.

\section{Gut microbiota transfer experiment}

As previously described [16], Each mouse was fed with streptomycin (20 mg dissolved in $100 \mu \mathrm{l}$ PBS solution). Then all mice were divided into two groups. One group received the gut microbiota from WT mice, and the other received the gut microbiota from Dock $2^{-/-}$mice from the previous cohort. WT and Dock $2^{-/-}$mice $(\mathrm{n}=3-4$ for each genotype) were selected as donors. Fresh feces were taken from 9 to 10 AM every day (To ensure that the feces of the two type donors were of the similar quality each time), and the fecal transplantation were completed within $1 \mathrm{~h}$ after the feces were taken. Specifically, the same weights of feces were homogenized in $0.05 \%$ cystine $\mathrm{HCl}$ PBS and each mouse received $100 \mu \mathrm{l}$ supernatant every other day. After six consecutive times, mice were infected with $C$. rodentium. Fecal samples were collected before and after streptomycin treatment and after 6 times of gut microbiota transfer.

\section{Antibiotic treatment experiment}

As previously described [17], antibiotic cocktail (1 mg/ $\mathrm{ml}$ ampicilln, $1 \mathrm{mg} / \mathrm{ml}$ metronidazole, $1 \mathrm{mg} / \mathrm{ml}$ neomycin, and $0.5 \mathrm{mg} / \mathrm{ml}$ vancomycin) was added into the drinking water for 4 weeks. One day after the termination of antibiotic treatment, the mice were infected with C. rodentium.

\section{Citobacter rodentium infection}

C. rodentium was cultured overnight and then sub-cultured into longitude phase. The mice were orally administrated with C. rodentium at the dose of $1 \times 10^{10} \mathrm{CFU} /$ mouse as previously described [6]. To determine the bacterial load, feces were homogenized and serially diluted 
in PBS, then the diluent was plated onto McConkey agar plates for CFU counting. After infection, mice were sacrificed. Colons were collected and colon lengths were measured.

\section{$16 \mathrm{~S}$ rRNA gene sequencing and data processing of gut microbiota}

Genomic DNA from feces was extracted using fecal DNA extraction kit. The construction of high-throughput sequencing library and sequencing based on Illumina miseq platform were completed by GENEWIZ (Suzhou, China). A series of PCR primers were used to amplify two highly variable regions (V3 and V4) of prokaryotic $16 \mathrm{~S}$ rDNA. The upstream primers contained the sequence "CCTACGGRRBGCASCAKVGAAT" and the downstream primers contained the sequence "GGACTA CNVGGGTWTCTAATCC". In addition, the end of $16 \mathrm{~S}$ rDNA PCR product was inserted with an "Index" connector by PCR to facilitate NGS sequencing. The quality of the library was detected by Agilent 2100 Biological Analyzer (Agilent Technologies, Palo Alto, CA, USA), and the library concentration was detected by Qubit 2.0 Fluorometer (Invitrogen, Carlsbad, CA). After the DNA library was mixed, PE250/300 double-ended sequencing was performed according to the instruction manual of Illumina miseq (Illumina, San Diego, CA, USA), and the sequence information was read by Miseq Control Software (MCS) of Miseq.

Analysis of $16 \mathrm{~S}$ rRNA gene sequencing data generated in this study was performed according to the sequencing data processing steps as described in [18].

The differences in bacteria among various groups were analyzed by a variety of analysis methods, including heatmaps, Anosim, Metastasis, and LEfSe analysis.

\section{Analysis of inter group differences in a diversity index}

This is based on the $\alpha$ diversity index table, using $\mathrm{R}$ language to construct the box chart, which can show the maximum and minimum values, median and abnormal values of $\alpha$ diversity index of each group, and also can directly reflect the diversity degree between groups. It mainly includes Chao1 analysis and Shannon analysis. This analysis uses the Chaol algorithm to estimate the number of OTUs in the samples, which is often used to evaluate the total number of species in ecology. Shannon analysis was used to estimate microbial diversity in samples.

\section{Anosim analysis (analysis of similarities)}

Similarity analysis is a nonparametric test, which is used to test whether the difference between groups (two or more groups) is significantly greater than that within the group to judge whether the grouping is meaningful. The input data for the ANOSIM test were from the normalized OTU tables. The main reference values are $R$ value and $P$ value. $R$ : $R$ value range $[-1,1]$, the actual result is generally $[0,1]$. $R$ value close to 0 means that there is no significant difference between groups and within groups, and $R$ value close to 1 means that the difference between groups is greater than the difference within groups.

\section{Sibling mice experiment}

Our mouse breeding cages were $D o c k 2^{+/-}$mice and $D o c k 2^{+/-}$mice. The offspring from these breeding cages contained Dock $2^{+/+}(\mathrm{WT})$ mice and Dock $2^{-/-}$mice. In this way, these two genotype mice had the same parents and were reared in the same environments starting from their birth. These mice were fed in the same cages until the age of 4 weeks old and fecal samples were collected. After that, mice were separated according to their genotype. At the age of 10 weeks old, fecal samples of mice were collected again.

\section{Histology}

The colon tissue was fixed in $10 \%$ formalin, embedded in paraffin, sectioned $(5 \mu \mathrm{m})$ and stained with hematoxylin and eosin $(\mathrm{H} \& \mathrm{E})$. The sections were scored by the pathologist blindly based on the degree of inflammation, edema, hyperplasia, colon injury, and crypt length as previously described [19]. According to the progressive score, the degree of lesion was mainly determined by the depth of infiltration, the number of inflammatory cells and the degree of crypt damage. The score of inflammation range and degree: normal $=0$; mild $=1$; moderate $=2$; severe $=3$; the score of inflammation infiltration: normal $=0 ; \quad$ mucosa $=1 ;$ submucosa $=2$; full layer $=3$; crypt damage degree: normal $=0$; one third of basement crypt was destroyed $=1$; two thirds of basement crypt was destroyed $=2$; only complete surface epithelium $=3$; all crypt and epithelium were destroyed $=4$. The highest score is 10 .

\section{Statistical analysis}

GraphPad prism 8.0 was used for data analysis. The data was represented by mean \pm standard error (SEM). $P$ value was calculated by ANOVA or Student $t$ test. ${ }^{*} P<0.05$ was considered as significantly different. The results of $16 \mathrm{~S}$ rRNA sequencing were analyzed by nonparametric statistical analysis. Identification of significant taxa between groups was performed using both Metastats [20] and LEfSe [21] software. 


\section{Results}

Dock2 regulates the gut microbiota and affects the host susceptibility to Citrobacter rodentium infection

Female WT mice were cohoused or not cohoused with Dock $2^{-/-}$mice for 4 weeks and then infected with $C$. rodentium (Fig. 1A). Five days after infection, there was no difference in $C$. rodentium load of WT mice with or without cohousing. However, WT mice cohoused with Dock $2^{-/-}$mice had significantly more C. rodentium load than those not cohoused at 10, 14, and 17 days after infection (Fig. 1B). Consistent with this result, the cohoused WT mice had shorter colon lengths than those not cohoused at 21 days after infection (Fig. 1C). In contrast, no difference in $C$. rodentium load was identified in Dock $2^{-/-}$mice with or without cohousing throughout the experiment. These results indicated that WT and Dock $2^{-/-}$mice had differences in composition of gut microbiota, and WT mice might obtain gut microbiota from Dock $2^{-/-}$mice by co-housing, which increased their susceptibility to $C$. rodentium infection.

In order to further test whether the difference in gut microbiota between WT and Dock $2^{-/}$mice affects the host susceptibility to $C$. rodentium infection, we used a direct gut microbiota transfer experiment as previously described (Fig. 2A) [16]. On days 4, 7, 10, and 14 after $C$. rodentium infection, there was no difference in the $C$. rodentium load of WT mice between transferred with WT mouse microbiota and with Dock $2^{-/-}$mouse microbiota, indicating that Dock $2^{-/-}$mouse gut microbiota had no significant effect on $C$. rodentium load in early stage of infection. However, on day 21 after infection, the $C$. rodentium load of WT mice transferred with Dock $2^{-/-}$mouse microbiota was significantly higher (Fig. 2B). Consistently, the colon lengths of WT transferred with Dock $2^{-/-}$mouse microbiota were significantly shorter than those transferred with WT mouse microbiota controls (Fig. 2C). Furthermore, H \& E staining results showed that the colonic tissues of WT mice transferred with Dock $2^{-/-}$mouse microbiota group had more serious crypt hyperplasia and more inflammatory cell infiltration than those in controls (Fig. 2D, E). The results collectively supported that WT and Dock $2^{-1-}$ mice had difference in gut microbiota, which might affect the host susceptibility to $C$. rodentium infection.

To further certify the results, antibiotic treatment experiment was performed (Fig. 3A). Our results showed that both WT and Dock $2^{-/-}$mice treated with antibiotic could not clear $C$. rodentium on days 5, 10 and 17 day after infection, which indicated that removal of gut microbiota reversed the difference of bacterial loads (Fig. 3B-D). These results suggest that the gut microbiota was critical for the host to eliminate C. rodentium infection, and the host susceptibility to

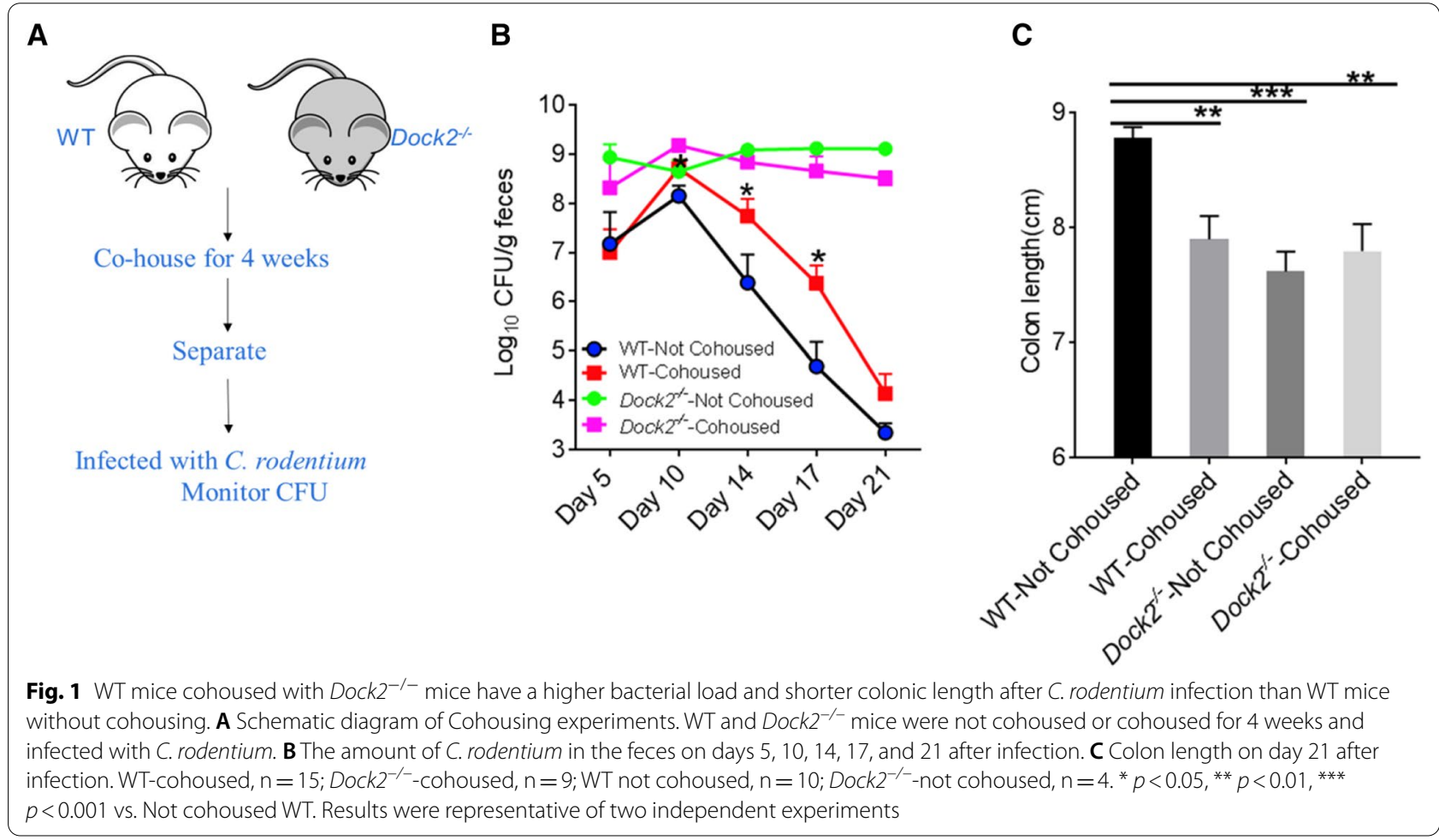


A

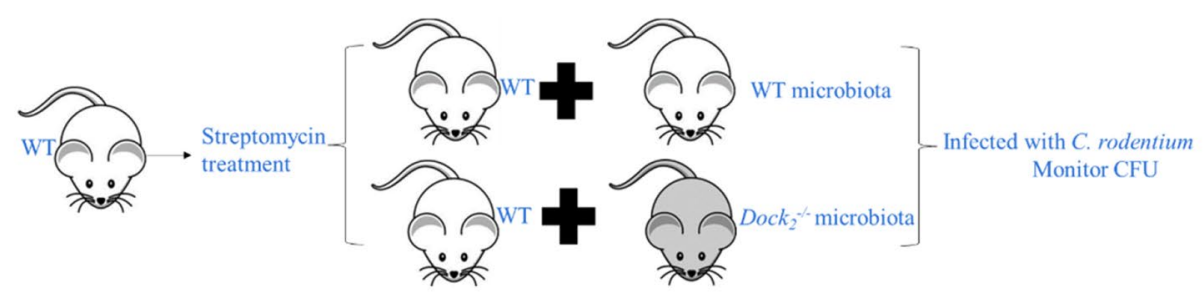

B

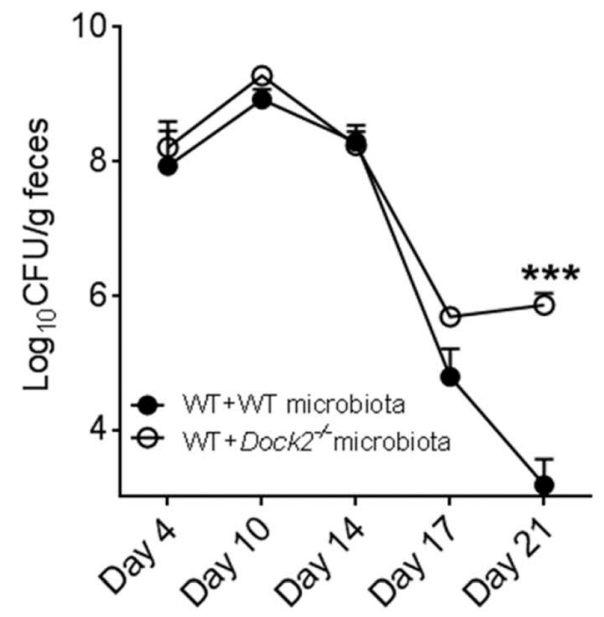

C
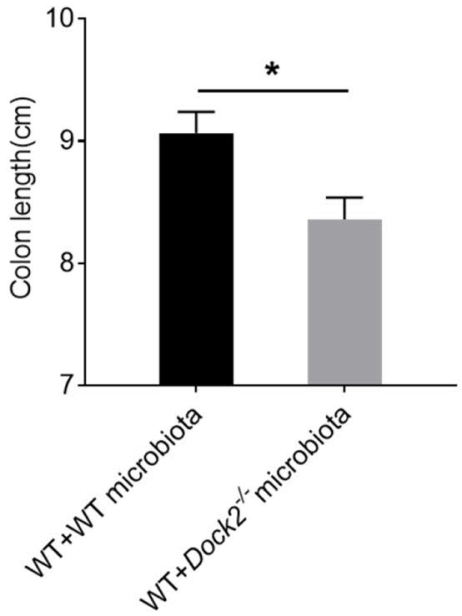

D
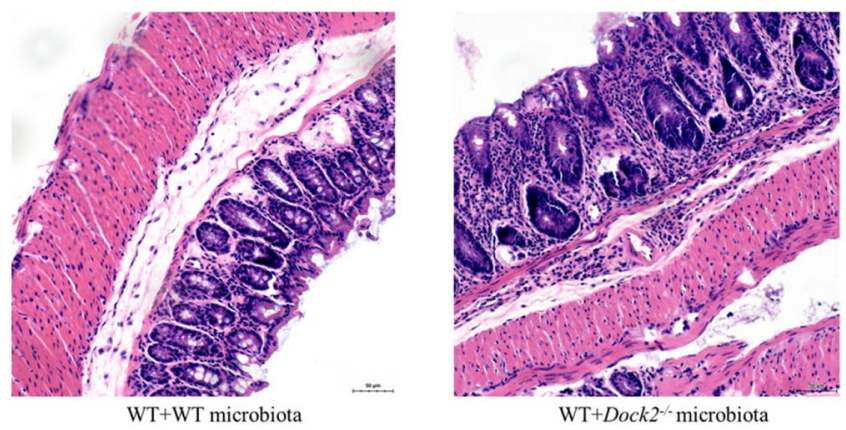

E

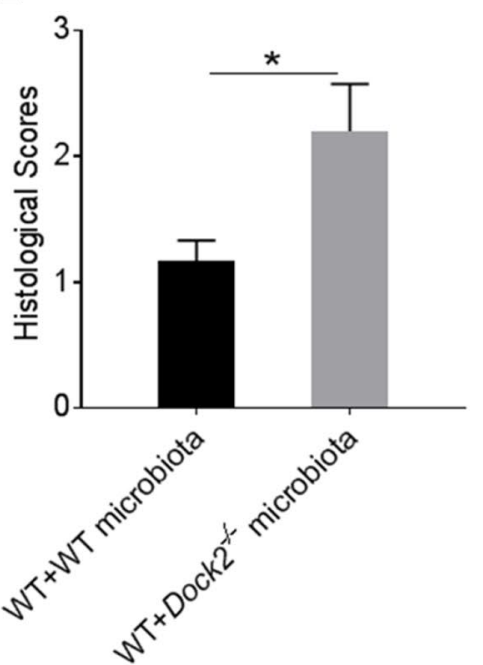

Fig. 2 WT mice transferred with gut microbiota of Dock $2^{-/-}$mice have more bacterial load, shorter colon length, and more severe intestinal inflammation than WT mice transferred with WT mouse gut microbiota. A Schematic diagram of gut microbiota transfer experiments. WT mice were treated with streptomycin and transferred with WT or Dock $2^{-/}$mouse gut microbiota, then infected with C. rodentium. Mouse feces were collected to measure bacterial load on days 4, 10,14, 17, and 21. Mice were sacrificed on day 21 and colon lengths were measured. B C. rodentium bacterial load. C Colon length on day 21. D, E H \& E staining photograph of colonic tissues. Scale bar $50 \mu \mathrm{m}$. WT mice $n=6 ;$ Dock $2^{-1-}$ mice $n=5$. ${ }^{*}$ $p<0.05,{ }^{* * *} p<0.001$. Data were presented as mean \pm SEM. Results were representative of two independent experiments 
A

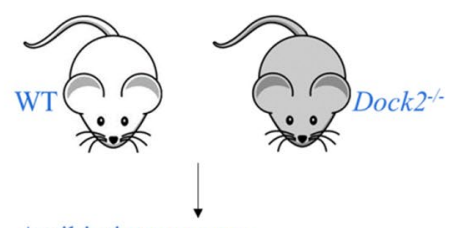

Antibiotic treatment

Infected with $C$. rodentium

Monitor CFU

C

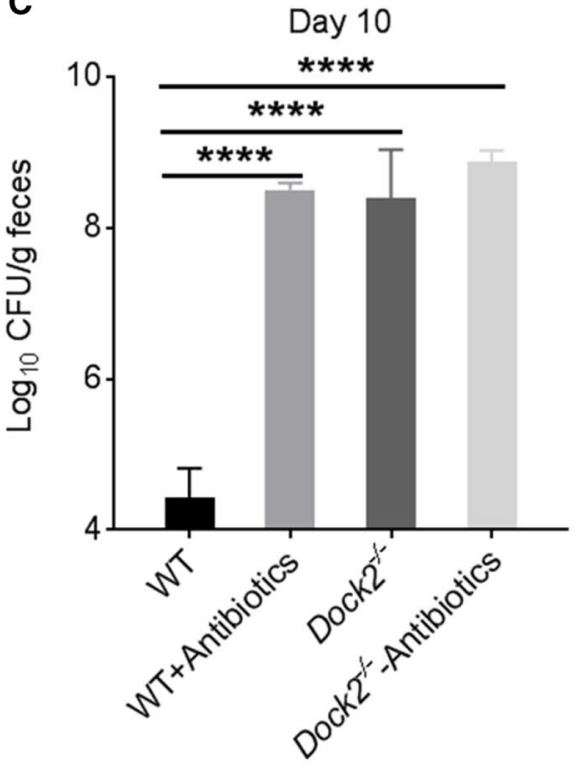

B

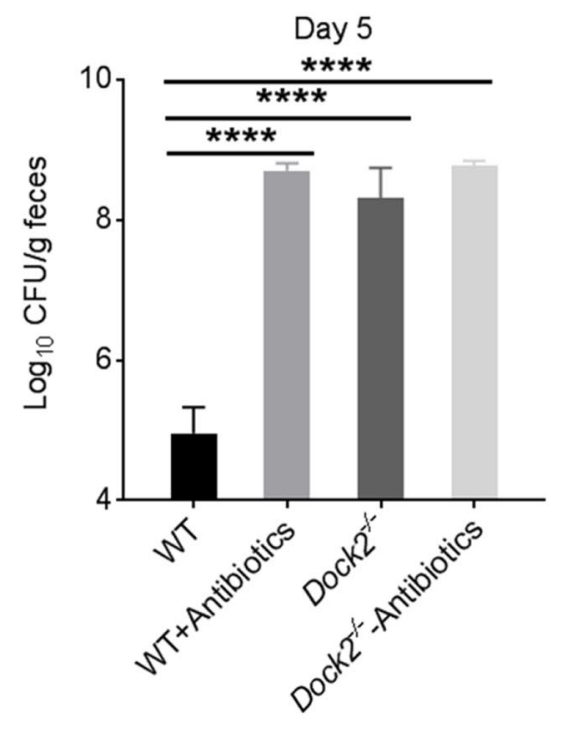

D

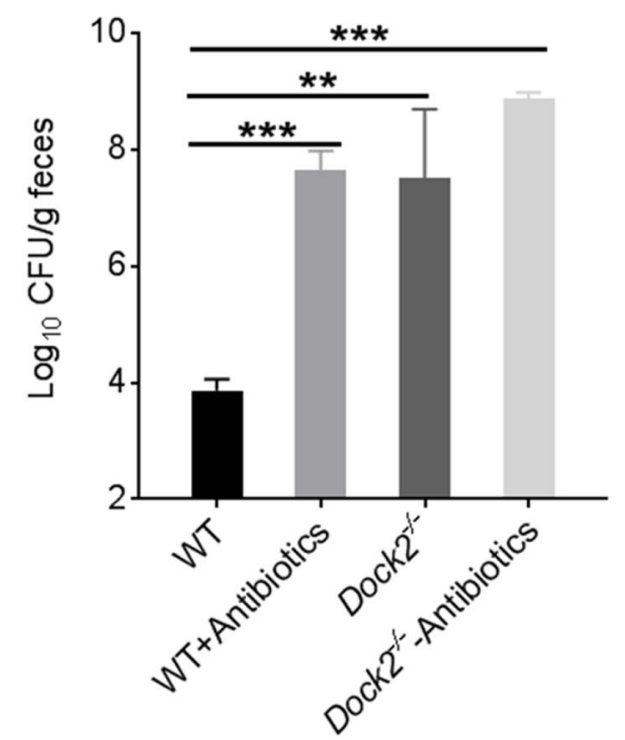

Fig. 3 Antibiotic treatment reversed the difference in the host susceptibility of WT and Dock $2^{-/}$mice to C. rodentium infection. A Schematic diagram of antibiotic treatment experiments. WT and Dock $2^{-1-}$ mice were infected with C. rodentium after 4 weeks of antibiotic treatment. Feces were collected on days 5 (B), $10(\mathbf{C})$, and 17 (D) for C. rodentium load testing. At day 5, WT, $n=7 ; W T+$ Antibiotics, $n=4 ; D o c k 2^{-/-}, n=6$;

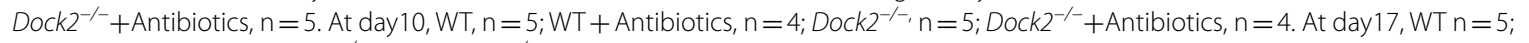
WT + Antibiotics $n=4 ;$ Dock $2^{-1-} n=3$; Dock2 ${ }^{-1-}+$ Antibiotics $n=3 .{ }^{* *} p<0.01,{ }^{* * *} p<0.001,{ }^{* * *} p<0.0001$ vs. WT. Data were presented as mean \pm SEM. Results were representative of two independent experiments

C. rodentium infection was closely associated with the composition of gut microbiota.

The difference of gut microbiota between sibling WT and $D o c k 2^{-1-}$ mice.

We selected sibling WT and Dock $2^{-/}$mice, produced by mating Dock $2^{+/-}$and Dock $2^{+/-}$mice. The gut microbiota of sibling WT and Dock $2^{-/}$-mice were analyzed by $16 \mathrm{~S}$ rRNA gene sequencing. Heat map results showed that Dock2 $2^{-/-}$mice possessed more abundance in Helicobacter, Roseburia and Lachnoclostridium, while less abundance in Prevotellaceae-NK3B31-group compared to WT mice (Fig. 4A).

Our alpha diversity analysis based on Chao1 and Shannon indices showed that there is no significant difference in the species richness and diversity of gut microbiota between the sibling WT and Dock2-/- mice when 
examined at 4 weeks old of age and 10 weeks old of age (6 weeks after cage division), respectively (Fig. 4B and Additional file 1: Figure S1). Anosim test results showed no significant difference in the composition of gut microbiota between WT and Dock $2^{-/-}$mice at 4 weeks of age (Fig. 4C). This could be explained by the fact that WT mice and Dock $2^{-/-}$mice were cohoused before 4 weeks of age. Anosim test results for WT mice between 4 weeks and 10 weeks of age were $R=0.102$ and $p=0.033$, indicating that there is a significant but slight gut microbial composition difference in the WT mice before and after cage division (Fig. 4D). Anosim test results for Dock $2^{-/-}$ mice between 4 and 10 weeks of age were $\mathrm{R}=0.172$ and $p=0.005$, suggesting that the gut microbiota of Dock $2^{-/-}$ mice before and after cage division was also significantly different (Fig. 4E). Moreover, the composition of gut microbiota in WT and Dock2 $2^{-/}$mice at 10 weeks old of age was significantly different according to Anosim test (Fig. 4F). The results showed that the gut microbiota became different after cage division possibly due to different genotypes.

We next analyzed the gut microbiota difference between sibling WT and Dock $2^{-/-}$mice after cage division. Metastats test results showed that Dock $2^{-/-}$mice had higher contents of Roseburia and Helicobacter, lower contents of Allobaculum, Bacteroidales-S24-7-groupunclassified, and Prevotellaceae -NK3B31- group (Fig. 4G-K). The LEfSe test chart showed that the gut microbiota difference between WT and Dock $2^{-/-}$mice was prevotellaceae-NK3B31-group, proteobacteria, Epsilonproteobacteria, Helicobacteraceae, Campylobacterales, and Helicobacter (Fig. 4L, M). In short, at 6 weeks after cage division, Dock $2^{-/}$mice had decreased Prevotellaceae-NK3B31-group and increased Helicobacter in the gut microbiota compared to WT mice.

\section{The difference of gut microbiota before and after} co-housing

As described in Fig. 1, WT mice co-housed with Dock $2^{-/-}$mice got certain gut microbiota from Dock $2^{-/-}$ mice and reduced the resistance to $C$. rodentium infection. To detect the specific exchanged gut microbiota, the analysis of $16 \mathrm{~S}$ rRNA gene sequencing was performed. The results showed that WT mice cohoused with Dock $2^{-/-}$mice had increased Rikenellaceae-RC9gut-group and reduced Lactobacillus and Desulfovibrio
(Fig. 5A). Alpha diversity analysis demonstrated that there was no significant difference in the richness and diversity of gut microbiota in both the WT and Dock $2^{-/-}$ mice when they were each compared before and after the co-housing experiment (Fig. 5B and Additional file 1: Figure S2). The Anosim test, on the other hand, showed no significance difference in the gut microbial profiles of WT mice before and after co-housing (Fig. 5C). However, Metastats test results showed that WT mice cohoused with $D o c k 2^{-/-}$mice had higher load of Alisipes and Rikenellaceae-RC9-gut-group and lower load of Lactobacillus (Fig. 5D-F).

LEfSe test results showed that co-housing reduced the relative abundance of Deltaproteobacteria, Dessulfovibrionaceae, Dessulfovibrionales, LachnospiraceaeUCG006, ASF356, Lactobacillaceae, Lactobacillus Bacilli, Deferribacteraceae, Deferribacterales, Defferribacteres, Mucispirillum, and Anaerotruncus, while reduced the relative abundance of Eubacterium-xylanophilum-group, Streptococcaceae, Rickettsiales, Lactococcus, Streptococcus, Mitochondria, A2 and Runinococcaceae-UCG-009 (Fig. 5G, H). Based on those analysis, we inferred that WT mice cohoused with Dock $2^{-/-}$mice possessed lower abundance of Lactobacillus, which might be associated with increased host susceptibility to $C$. rodentium infection.

\section{Gut microbiota analysis in transfer experiment}

In order to determine specific gut microbiota relating to the host susceptibility to $C$. rodentium infection, we performed $16 \mathrm{~S}$ rRNA gene sequencing after the gut microbiota transfer experiment as shown in Fig. 2. The results showed that WT mice receiving Dock $2^{-1}$ mouse microbiota (FA-K) had higher levels of bacteria including Helicobacter, Dubosiella, Parabacteroides, and lower levels of bacteria including Prevotellaceae-NK3B31group, g-Muribaculum- Unclassified, LachnospiraceaeNK4A136-group, Lachnospiraceae-Unclassified (Fig. 6A). Based on the alpha diversity analysis, it was shown that the gut bacterial species diversity, not species richness, was significantly affected in the mice involved in the fecal transplant experiment. After antibiotic treatment and prior to the fecal transplant procedure, both FB-W and FB-K mice had a significant $(\mathrm{p}=0.037)$ and a marginally significant $(\mathrm{p}=0.07)$ reduction in microbial

\footnotetext{
(See figure on next page.)

Fig. 4 16 S rRNA gene sequencing results of gut microbiota of siblings of WT and Dock2 $2^{-/}$mice. Feces were collected from WT and Dock2 ${ }^{-/-}$ mice before and after cage division (4 weeks and 10 weeks). DNA was extracted, and submitted to $16 \mathrm{~S}$ rRNA gene sequencing analysis. A The heat map of the gene level based on the 30 most abundant OTUs in the sequencing results; B The microbiota abundance index Chao 1 diversity analysis; C-F Anosim test results; $\mathbf{G}-\mathbf{K}$ Metastats test results; L, $\mathbf{M}$ LEfSe test results; $\mathbf{A}$ represented the siblings of WT mice before cage division, $\mathbf{B}$ represented the siblings of Dock $2^{-I}$ mice before cage division, $\mathbf{C}$ represented siblings of WT mice at 6 weeks after cage division, and $\mathbf{D}$ represented the siblings of Dock $2^{-1-}$ mice at 6 weeks after cage division. ${ }^{*} p<0.05,{ }^{* *} p<0.01$, ns, not significantly different
} 


\section{A}

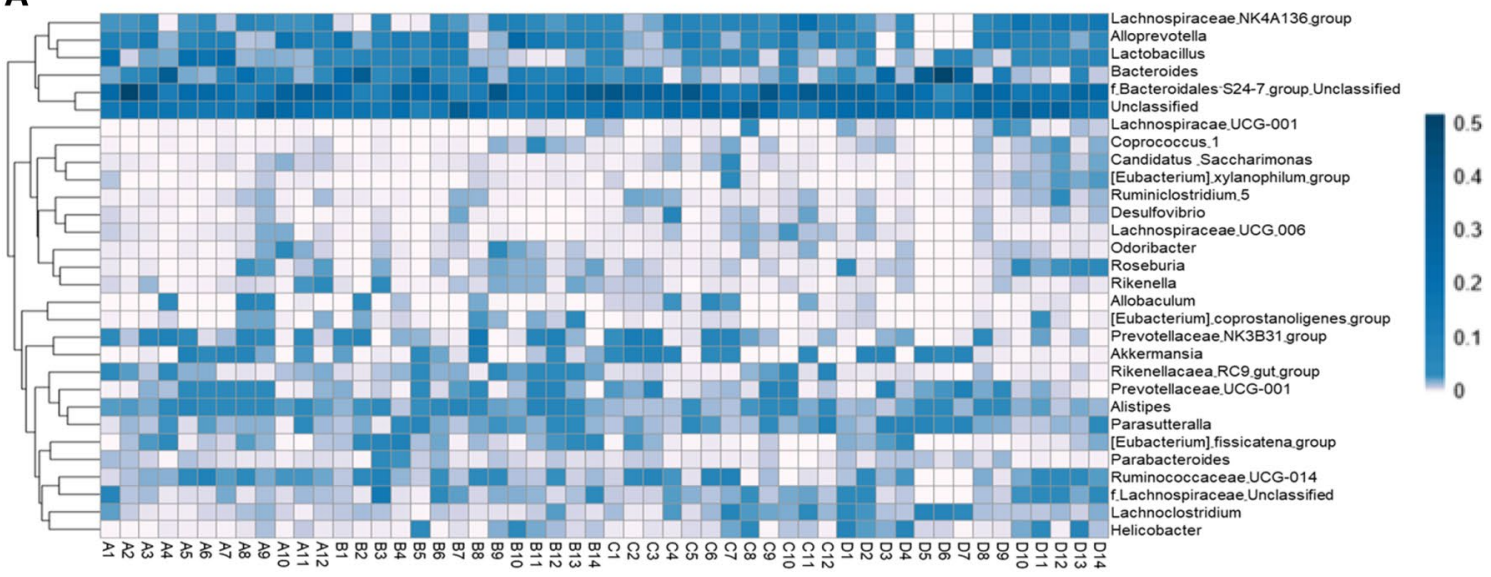

B

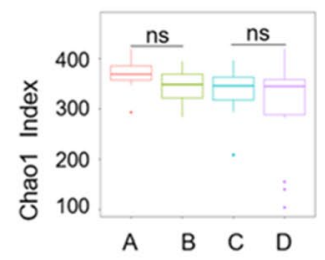

$\mathbf{F}$

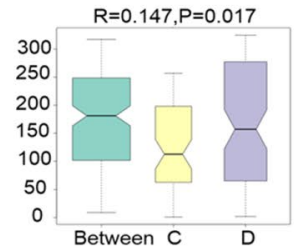

I

$\mathbf{L}$
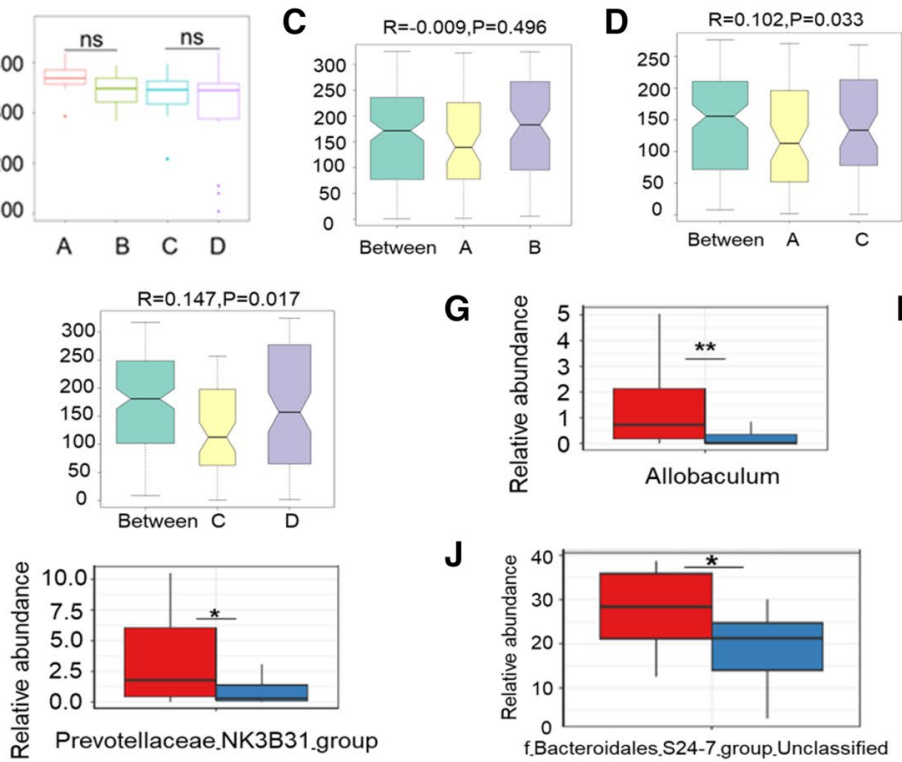

G

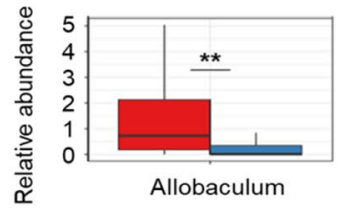

J

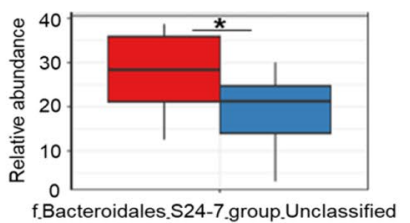

E $\quad \mathrm{R}=0.172, \mathrm{P}=0.005$

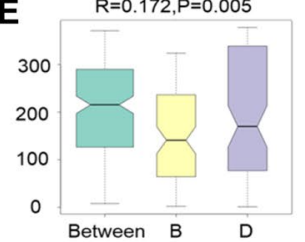

H

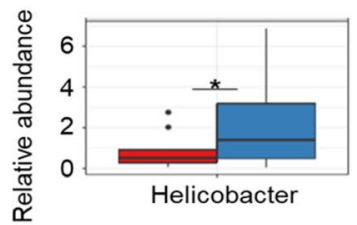

K

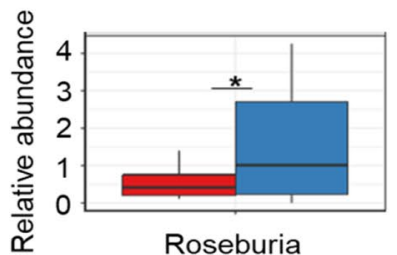

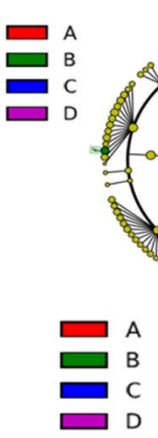

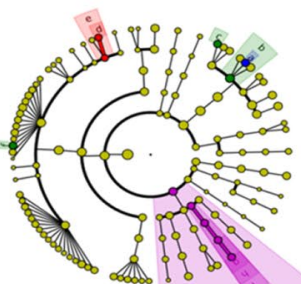

a:g.Prevotellaceae_NK3B31_group

$\checkmark$ b.f.Prevotellaceae

c:g.Rikenellaceae RC9 gut group

d:g.Candidatus Arthromitus

e:f.Clostridiaceae-1

f:g.Eubacterium-fissicatena-group

g:g.Helicobacter

- hif Helicobacteraceae

i:o.Campylobacteria

j:c.Epsilonproteobacteria

\section{M}
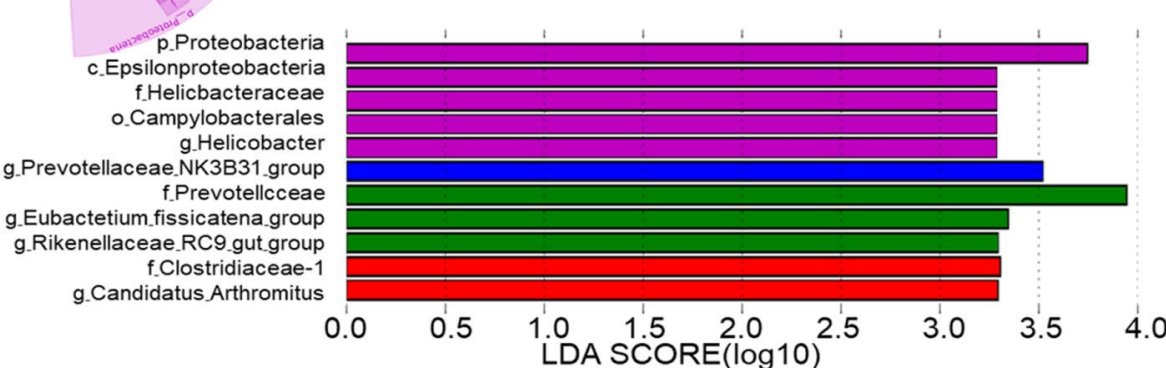

Fig. 4 (See legend on previous page.) 
species diversity, respectively, when compared to that of the treatment naïve WT mice based on Shannon anlaysis but not Chao1 analysis(Fig. 6B and Additional file 1: Figure S3). After fecal transplant, a significant restoration of microbial species diversity was observed only in mice receiving the WT fecal material (FA-W group) $(\mathrm{p}=0.019)$, but not in those which had the Dock $2^{-1-}$ fecal material (FA-K group) (Fig. 6C). The Anosim test result further demonstrated that the gut microbiota of the FA-K and FA-W groups are significantly different from each other $(R=0.393, p=0.005)$, as depicted in Fig. 6D.

The Metastats test showed that WT mice receiving Dock $2^{-/-}$mice gut microbiota had lower levels of bacteria including Prevotellaceae-NK3B31-group, Rikenellaceae-RC9-gut-group [Eubacterium]-fissicatena-group and higher levels of bacteria including Dubosiella and Parabacteroides (Fig. 6E-I). The relative abundance between groups was further analyzed by LEfSe test, which showed that WT mice (FA-W) possessed Prevotellaceae-NK3B31-group; Rikenellaceae-RC9- gut-group; Muribaculum; while Dock $2^{-/}$bacteria (FA-K) mice possessed Dubosiella; Parabacteroides; Tannerellaceae; Erysipelotrichaceae; Erysipelotrichales; Erysipelotrichia (Fig. 6J, K). Those analysis showed that WT mice transferred with $D o c k 2^{-/-}$mouse gut microbiota had fewer Prevotellaceae-NK3B31-group than the WT mice transferred with WT gut microbiota, which was consistent with the results of the gut microbiota in the siblings experiment.

In summary, Dock $2^{-/-}$mice have fewer PrevotellaceaeNK3B31-group, Lactobacillus and higher Helicobacter than the WT mice. Prevotellaceae-NK3B31 -group and Lactobacillus may be beneficial for the host to defend against $C$. rodentium infection, while Helicobacter may aggravate the host susceptibility to $C$. rodentium infection.

\section{Discussion}

Our previous study showed that Dock $2^{-/-}$mice were more susceptible to colitis induced by $C$. rodentium infection than WT mice [6]. However, the exact mechanism by which Dock2 protects host from enteric bacterial infection or colitis has not been described. In this study, we demonstrated that Dock2 plays a key role in the response to $C$. rodentium infection through regulating gut microbiota by co-housing, fecal microbiota transfer and antibiotic treatment methods. Furthermore, using $16 \mathrm{~S}$ rRNA gene sequencing analysis, we showed that Dock2 induced the increase in the abundance of prevotellaceae-NK3B31-group and Lactobacillus and the decrease in the abundance of Helicobacter.

The gut microbiota is a key player in mammalian physiology and participates in the protection against $C$. rodentium infection. Studies have shown that gene deficiency could alter the composition of gut microbiota and affect the host susceptibility to intestinal pathogens. For instance, it has been reported that alteration in the composition of gut microbiota caused by Nod2 deficiency gave rise to a reversible risk of colitis in mice, while reciprocal microbiota transplantation reduced disease risk [22]. A study showed that Nlrp6 deficiency in mouse colonic epithelial cells resulted in altered fecal microbiota characterized by expanded representation of bacterial phyla Bacteroidetes (Prevotellaceae) and TM7 [23]. Another study showed significant changes in the abundance of the Firmicutes and Bacteroidetes phyla, when comparing Caspase- $1,-7$ and -3 knockout mice to WT mice [24]. Furthermore, the microbiota of Card 9 deficiency mice contributed to the decreased host resistance to $C$. rodentium infection. These lines of evidence implied that the composition of the gut microbiota could be regulated by various genes [24]. Consistent with these findings, we showed that Dock2 deficiency has significant consequences on the composition of gut microbiota characterized by the decrease in Prevotellaceae-NK3B31group and Lactobacillus and the increase in Helicobacter, which was linked to the enhanced vulnerability to $C$. rodentium infection.

Lactobacillus represents a source of lactic acid-producing probiotic bacteria. Reported benefits of lactobacilli include their ability to activate the host immune system, prevent the duration and intensity of diarrheal episodes, enhance colonization resistance, and produce bacteriocins (pathogen inhibitory compounds) [25]. It was found that expansion of gut Lactobacilli communities inhibited the amplification of $\gamma$ - Proteobacteria and actinomycetes, improved the mucosal barrier function, reduced the

\footnotetext{
(See figure on next page.)

Fig. $516 \mathrm{~S}$ rRNA gene Sequencing results of WT and Dock $2^{-/}$mouse gut microbiota before and after cohousing. Feces were collected from WT and Dock $2^{-1-}$ mice before and after cohousing, and submitted to DNA extraction for $16 \mathrm{~S}$ rRNA gene sequencing analysis. A Genus heat map based on the 30 most abundant OTUs in sequencing results; B The microbiota abundance index Chao 1 diversity analysis; C Anosim test results; D-F Metastats test results; G, H LEfSe test results; CB-W represented WT mice before cohousing, CB-K represented Dock ${ }^{-/-}$mice before cohousing, CA-W represented WT mice 4 weeks after cohousing, and CA-K represented Dock $2^{-/}$mice after cohousing. ${ }^{*} p<0.05,{ }^{* *} p<0.01$, ns, not significantly different
} 


\section{A}

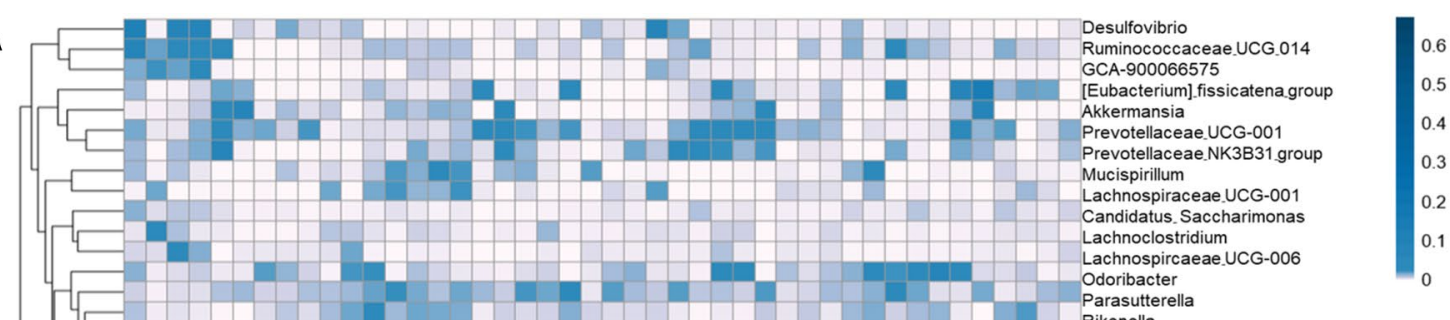
arasutterella

Rikenella

(n)

Ruminiclostriidium

fClostridiales vadinBB60 group Unclassified

Parabacteroides

Prevotellaceae.Unclassified

Muribaculum

Rikenellaceae RC9 gut group

actobacillus
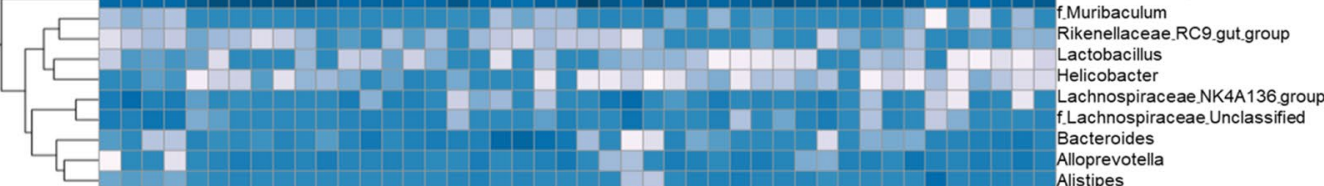

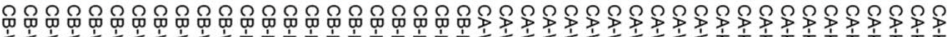

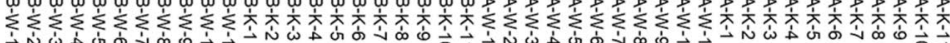

B

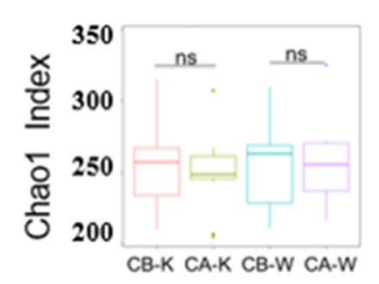

Group
CA-W
CB-W
C

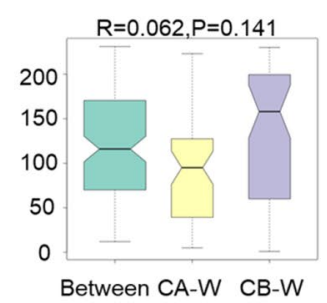

E

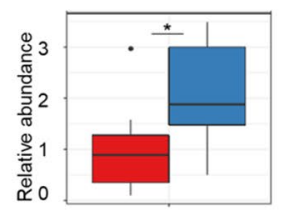

Lactobacillus
D

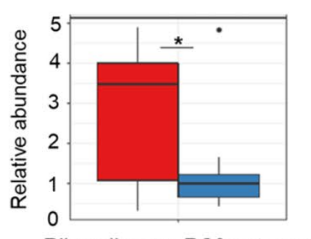

Rikenellaceae-RC9-gut-group

$\mathbf{F}$

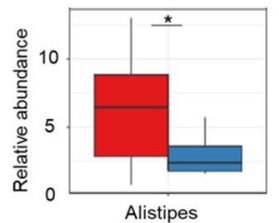

G

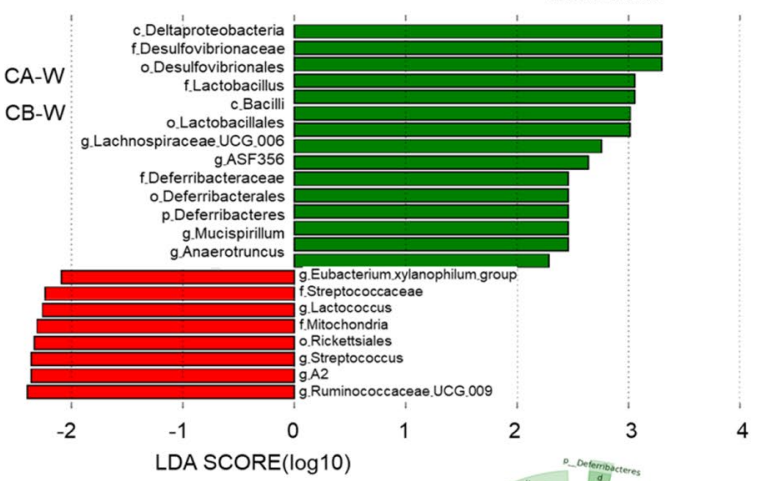

H

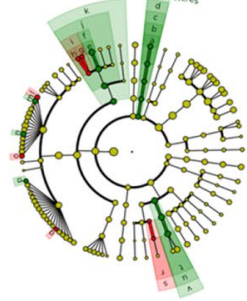

$\square B-W$
CB-W

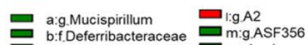

c:o.Deferribacterales ${ }_{\text {n:g.Lachnospiraceae UCG } 006}$

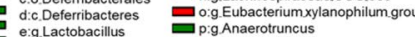

e:g.Lactobacillus
iff.tactobacillaceae
q:g.R.Ruminocococcaceae.UCG.009

g:g.Lactococcus
h:g.Streptococcaceae Mitochondria
s:o.Rickettsiales

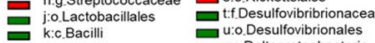

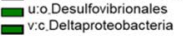

Fig. 5 (See legend on previous page.) 
production of infectious colonic crypt hyperplasia and tissue inflammatory factors, thus enhancing the resistance of mice to $C$. rodentium infection [26]. Numerous studies demonstrated that the Lactobacilli-enriched commensal gut microenvironment protects against $C$. rodentium infection and colitis [27]. Therefore, the significant decrease of Lactobacillus in the Dock $2^{-/-}$mice could mean that they benefit less from the positive effects of Lactobacillus, which may render the host more susceptible to C. rodentium infection. Helicobacter pylori is a gram-negative pathogenic bacterium and its infection in humans has been a challenge due to a higher incidence rate of the disease $[28,29]$. H. pylori infection is implicated in the pathogenesis of gastritis, gastric ulcer, gastric cancer, gastric mucosa-associated lymphoid tissue (MALT) lymphoma, IBD, and CRC [30]. The analysis of the gut microbiota in IBD patients showed the increase in Proteobacteria phylum [31]. Studies have shown that Helicobacter genus is prone to induce colitis in genedeficient mouse animal models [32]. Therefore, the significant increase of Helicobacter in the Dock $2^{-/}$mice suggested that these mice were more susceptible to $C$. rodentium than the WT mice.

There is an evidence suggesting that Prevotella is associated with opportunistic infections, such as periodontitis or bacterial vaginosis [33]. Prevotella has been implicated in rheumatoid arthritis [34], human immunodeficiency virus (HIV) infection [35], and IBD [34]. Expansion of intestinal Prevotella copri correlates with enhanced susceptibility to DSS-induced colitis [34]. Prevotella promote experimental colitis in mice, indicating that prevotella may be harmful.

However, Prevotella is also considered to be a common symbiotic bacterium due to its presence in healthy humans, including the mouth, gastrointestinal tract, urogenital tract, and skin. It was reported that the protection of mice from lethal colitis was associated with higher levels of bacteria from Bacteroidetes [36]. Prevotellaceae is a branch of Bacteroidates, which can promote the production of butyrate, a short-chain fatty acid (SCFA) [37]. SCFAs can enhance the expression of tight junction protein, maintain the integrity of epithelial barrier, and reduce the expression of proinflammatory cytokines in mucus [38]. Stachyose has been reported to improve the intestinal homeostasis in high-fat diet -fed mice by improving the bacterial diversity and the increases in the relative abundances of gut microbiota including prevotellaceae-NK3B31-group [39]. In addition, Prevotella induced the homeostasis of glucose by regulating gut gluconeogenesis [40]. These findings suggest the beneficial effects of Prevotella. In agreement with these studies, our results showed a significant decrease of PrevotellaceaeNK3B31-group in the Dock2 $2^{-/-}$mice, which indicated that these mice may have less SCFAs in their intestine and benefit less from the positive effects of Prevotella. Prevotella is a large genus with species diversity. Different species may exert different function. In addition, the role of Prevotella may depend on the experimental models and disease. Our present finding showed that the decrease of prevotellaceae-NK3B31-group in the Dock $2^{-1-}$ mice correlates with enhanced susceptibility to C. rodentium infection.

\section{Conclusions}

To date, we described a clear link between Dock2 deficiency and gut microbiota composition. Our results indicated that Dock2 deficiency should be added to the list of host genetic factors that may drive alterations in the gut microbiota, which in turn may promote intestinal disease. However, additional studies are needed in future work. For instance, the transfer of prevotellaceaeNK3B31 to Dock $2^{-1-}$ mice is required to verify its biological significance in the host defense against $C$. rodentium infection. Mechanistic details underlying how Dock2 regulates gut microbiota against $C$. rodentium infection also need to be further characterized.

\footnotetext{
(See figure on next page.)

Fig. 6 16 S rRNA Sequencing results of WT and Dock $2^{-/-}$gut microbiota before and after transfer to WT mice. WT mice were treated with streptomycin and then transferred with gut microbiota of WT or Dock $2^{-/-}$. Fecal samples were collected before and after gut microbiota transfer. DNA were extracted for $16 \mathrm{~S}$ rRNA gene sequencing analysis. A The heat map of the 30 most abundant OTUs in the sequencing results. B, C The microbiota abundance index shannon diversity analysis; $\mathbf{D}$ Anosim test result results. E-I Metastats test results. J, K LEfSe test results. AB represented mice before streptomycin treatment; FB-W represented WT mice before transfer, after which they were transferred with WT gut microbiota; FB-K represented for WT mice before transfer, after which they were transferred with Dock $2^{-1}$ - mouse gut microbiota. FA-W represented WT mice, to which the WT mouse microbiota was transferred; FA-K represented WT mice, to which the Dock ${ }^{-/-}$mouse microbiota was transferred. ${ }^{*} p<0.05$, ${ }^{* *}$ $p<0.01$, ns, not significantly different
} 
A
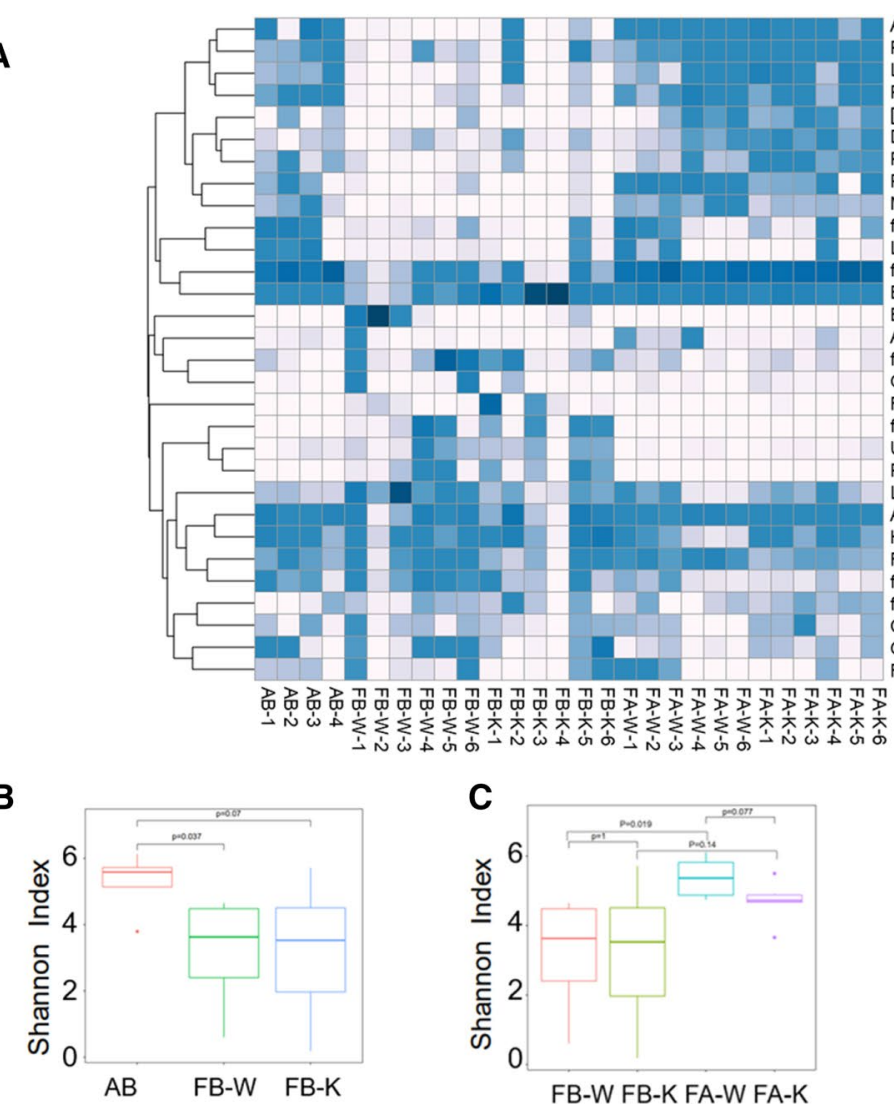

Alloprevotella

Parasutterella

Lachnoclostridium

[Eubacterium]_coprostanoligenes group

Dubosiella
Parabacteroides

Prevotellaceae.NK3B31 group

Muribaculum

fLachnospiraceae.Unclassified

Lachnospiraceae NK4A136 group

f-Muribaculaceae.Unclassified

Bacteroides

Enterococcus

f. Atopobiaceae Unclassified

GCA-900066225

f Mitochondria Unclassified

Unclassified Unclassified

Phaseolus_acutifolus.[tepary bean]

Lactobacillus

Alistipes

Helicobacter

Rikenellaceae RC9 gut group

f Ruminococcaceae Unclassified

funcultured bacterium Unclassified

Candidatus Sacchairimonas

Odoribacter

Ruminococcaceae UCG-014

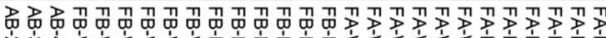

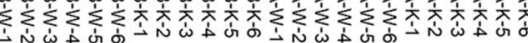

B
C

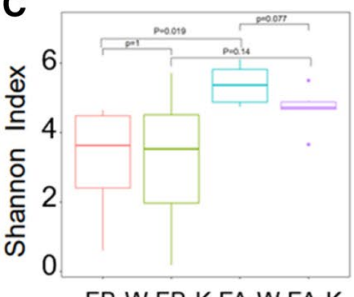

D

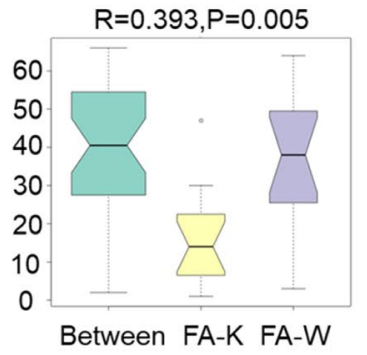

E

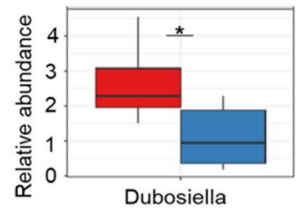

H

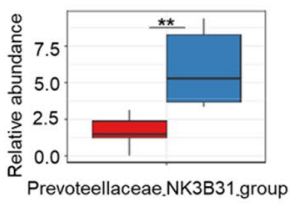

F

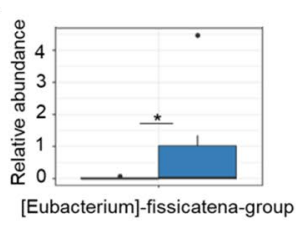

$\mathbf{G}_{\text {。 }}$

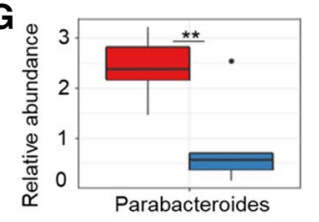

0.8

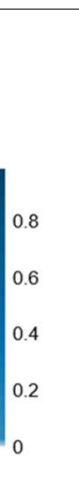




\section{Supplementary Information}

The online version contains supplementary material available at https://doi. org/10.1186/s13099-021-00449-x.

\section{Additional file 1: Figure S1. Shannon analysis of 165 rRNA gene} sequencing results from gut microbiota of siblings of WT and Dock $2^{-/-}$ mice. Shannon index in alpha diversity analysis of microbial community was shown. A represented the siblings of WT mice before cage division, $B$ represented the siblings of Dock ${ }^{-/-}$mice before cage division, $C$ represented siblings of WT mice at 6 weeks after cage division, and D represented the siblings of Dock $2^{-/}$mice at 6 weeks after cage division Figure S2. Shannon analysis of $16 \mathrm{~S}$ rRNA gene Sequencing results from WT and Dock $2^{-1-}$ mouse gut microbiota before and after cohousing. Shannon index in alpha diversity analysis of microbial community was shown. CB-W represented WT mice before cohousing, CB-K represented Dock $2^{-/-}$mice before cohousing, CA-W represented WT mice at 4 weeks after cohousing, and CA-K represented Dock $2^{-1-}$ mice after cohousing. Figure S3. chao 1 analysis of 16 S rRNA Sequencing results from WT and Dock $2^{-1-}$ gut microbiota before and after transfer to WT mice. Chao 1 index in alpha diversity analysis of microbial community was shown. $A B$ represented mice before streptomycin treatment; FB-W represented WT mice before transfer, after which they were transferred with WT gut microbiota; FB-K represented for WT mice before transfer, after which they were transferred with Dock ${ }^{-/-}$mouse gut microbiota. FA-W represented WT mice which received WT mouse microbiota; FA-K represented WT mice which received Dock $2^{-1-}$ mouse microbiota.

\section{Acknowledgements}

We are grateful to Dr. Yoshinori Fukui (Kyushu University, Japan), who provided Dock $2^{-/-}$mice used in the study.

\section{Authors' contributions}

$Y X$ performed experiments, data analysis and interpretation; $Y X, J C, B W$, TH drafted and revised the manuscript; LX performed part of experiments; ZL designed the study and revised the manuscript. All authors read and approved the final manuscript.

\section{Funding}

This work was supported by funds from Natural Science Foundation of Jiangxi Province (20151BAB205061 and 20171ACB20024), National Natural Science Foundation of China (31560260, 31960163), The Scientific and Technological Innovation Team Project of Gannan Medical University (TD201703) (to Zhiping Liu).

\section{Availability of data and materials}

The datasets used and/or analysed during the current study are available from the corresponding author on reasonable request.

\section{Declarations}

\section{Ethics approval and consent to participate}

This study was approved by the Ethics Committee of Gannan Medical University.

\section{Consent for publication}

All authors read and approved the submission of this manuscript.

\section{Competing interest}

The authors declare that they have no competing interests.

\section{Author details}

'Department of Nephrology, The First People's Hospital of Nankang District, Ganzhou, Jiangxi, China. ${ }^{2}$ Gannan Medical University, Ganzhou, Jiangxi, China. ${ }^{3}$ School of Basic Medicine, Gannan Medical University, Jiangxi, Ganzhou, China. ${ }^{4}$ Center for Immunology, Key Laboratory of Prevention and Treatment of Cardiovascular and Cerebrovascular Diseases, Ministry of Education, Gannan Medical University, Jiangxi, Ganzhou, China.
Received: 28 January 2021 Accepted: 4 August 2021

Published online: 14 August 2021

\section{References}

1. Torres J, et al. Crohn's disease. Lancet. 2017;389(10080):1741-55

2. Ungaro R, et al. Ulcerative colitis. Lancet. 2017;389(10080):1756-70.

3. Thaiss $C A$, et al. The microbiome and innate immunity. Nature. 2016;535(7610):65-74.

4. Eckmann L. Animal models of inflammatory bowel disease: lessons from enteric infections. Ann NY Acad Sci. 2006;1072:28-38.

5. Namekata K, et al. Dock GEFs and their therapeutic potential: neuroprotection and axon regeneration. Prog Retin Eye Res. 2014;43:1-16.

6. Liu Z, et al. DOCK2 confers immunity and intestinal colonization resistance to Citrobacter rodentium infection. Sci Rep. 2016;6:27814.

7. Mullineaux-Sanders C, et al. Citrobacter rodentium-host-microbiota interactions: immunity, bioenergetics and metabolism. Nat Rev Microbiol. 2019;17(11):701-15.

8. Kamada $\mathrm{N}$, et al. Regulated virulence controls the ability of a pathogen to compete with the gut microbiota. Science. 2012;336(6086):1325-9.

9. Hrdý J, et al. Lactobacillus reuteri 5454 and Bifidobacterium animalis ssp. lactis 5764 improve colitis while differentially impacting dendritic cells maturation and antimicrobial responses. Sci Rep. 2020;10(1):5345.

10. Ivanov II, et al. Induction of intestinal Th17 cells by segmented filamentous bacteria. Cell. 2009;139(3):485-98.

11. Atarashi $\mathrm{K}$, et al. Th17 cell induction by adhesion of microbes to intestinal epithelial cells. Cell. 2015;163(2):367-80.

12. Ryu SH, et al. The probiotic lactobacillus prevents Citrobacter rodentium-induced murine colitis in a TLR2-dependent manner. J Microbiol Biotechnol. 2016;26(7):1333-40.

13. Geiger TL, et al. Nfil3 is crucial for development of innate lymphoid cells and host protection against intestinal pathogens. J Exp Med. 2014;211(9):1723-31.

14. Desai MS, et al. A dietary fiber-deprived gut microbiota degrades the colonic mucus barrier and enhances pathogen susceptibility. Cell. 2016;167(5):1339-53.e21.

15. Man SM, et al. Critical role for the DNA sensor AIM2 in stem cell proliferation and cancer. Cell. 2015;162(1):45-58.

16. Willing $B P$, et al. Altering host resistance to infections through microbial transplantation. PLoS One. 2011;6(10):e26988.

17. Kuhn KA, et al. Bacteroidales recruit IL-6-producing intraepithelial lymphocytes in the colon to promote barrier integrity. Mucosal Immunol. 2018:11(2):357-68

18. Han Z, et al. Vertical variation of a black soil's properties in response to freeze-thaw cycles and its links to shift of microbial community structure. Sci Total Environ. 2018;625:106-13.

19. Zaki MH, et al. The NOD-like receptor NLRP12 attenuates colon inflammation and tumorigenesis. Cancer Cell. 2011;20(5):649-60.

20. White JR, Nagarajan N, Pop M. Statistical methods for detecting differentially abundant features in clinical metagenomic samples. PLoS Comput Biol. 2009;5(4):e1000352.

21. Segata N, et al. Metagenomic biomarker discovery and explanation. Genome Biol. 2011;12(6):R60.

22. Couturier-Maillard A, et al. NOD2-mediated dysbiosis predisposes mice to transmissible colitis and colorectal cancer. J Clin Invest. 2013:123(2):700-11.

23. Elinav E, et al. NLRP6 inflammasome regulates colonic microbial ecology and risk for colitis. Cell. 2011;145(5):745-57.

24. Brinkman BM, et al. Caspase deficiency alters the murine gut microbiome. Cell Death Dis. 2011;2(10):e220.

25. Kumar A, et al. Lactobacillus acidophilus counteracts inhibition of NHE3 and DRA expression and alleviates diarrheal phenotype in mice infected with Citrobacter rodentium. Am J Physiol Gastrointest Liver Physiol. 2016;311(5):G817-g826.

26. Jiminez JA, et al. Butyrate supplementation at high concentrations alters enteric bacterial communities and reduces intestinal inflammation in mice infected with Citrobacter rodentium. mSphere. 2017;2(4):e00243. 
27. Jiang $Y$, et al. Immunological mechanisms involved in probiotic-mediated protection against Citrobacter rodentium-induced colitis. Benef Microbes. 2016;7(3):397-407.

28. Bohr UR, et al. Identification of enterohepatic Helicobacter species in patients suffering from inflammatory bowel disease. J Clin Microbiol. 2004;42(6):2766-8.

29. Oliveira $A G$, et al. Isolation of Helicobacter pylori from the intestinal mucosa of patients with Crohn's disease. Helicobacter. 2006;11(1):2-9.

30. Inoue I, et al. Helicobacter pylori-related chronic gastritis as a risk factor for colonic neoplasms. World J Gastroenterol. 2014;20(6):1485-92.

31. Gophna U, et al. Differences between tissue-associated intestinal microfloras of patients with Crohn's disease and ulcerative colitis. J Clin Microbiol. 2006;44(11):4136-41.

32. Zhang L, et al. Natural colonization with Helicobacter species and the development of inflammatory bowel disease in interleukin-10-deficient mice. Helicobacter. 2005;10(3):223-30.

33. Larsen JM. The immune response to Prevotella bacteria in chronic inflammatory disease. Immunology. 2017;151(4):363-74.

34. Scher JU, et al. Expansion of intestinal Prevotella copri correlates with enhanced susceptibility to arthritis. Elife. 2013:2:e01202.

35. Dillon SM, et al. Gut dendritic cell activation links an altered colonic microbiome to mucosal and systemic T-cell activation in untreated HIV-1 infection. Mucosal Immunol. 2016;9(1):24-37.
36. Yue SJ, et al. Berberine treatment-emergent mild diarrhea associated with gut microbiota dysbiosis. Biomed Pharmacother. 2019;116:109002.

37. Esquivel-Elizondo $S$, et al. Insights into Butyrate Production in a Controlled Fermentation System via Gene Predictions. mSystems. 2017;2(4):e00051.

38. Ewaschuk JB, et al. Secreted bioactive factors from Bifidobacterium infantis enhance epithelial cell barrier function. Am J Physiol Gastrointest Liver Physiol. 2008;295(5):G1025-34.

39. Liu Y, et al. Regulatory effects of stachyose on colonic and hepatic inflammation, gut microbiota dysbiosis, and peripheral CD4(+)T cell distribution abnormality in high-fat diet-fed mice. J Agric Food Chem. 2019;67(42):11665-74.

40. De Vadder F, et al. Microbiota-Produced Succinate Improves Glucose Homeostasis via Intestinal Gluconeogenesis. Cell Metab. 2016;24(1):151-7

\section{Publisher's Note}

Springer Nature remains neutral with regard to jurisdictional claims in published maps and institutional affiliations.
Ready to submit your research? Choose BMC and benefit from:

- fast, convenient online submission

- thorough peer review by experienced researchers in your field

- rapid publication on acceptance

- support for research data, including large and complex data types

- gold Open Access which fosters wider collaboration and increased citations

- maximum visibility for your research: over $100 \mathrm{M}$ website views per year

At BMC, research is always in progress.

Learn more biomedcentral.com/submissions 